\title{
Hypoxia induces the translocation of glucose transporter 1 to the plasma membrane in vascular endothelial cells
}

\author{
Abdullah Al Mamun ${ }^{1,2}$, Hisaki Hayashi ${ }^{1}$, Aya Yamamura ${ }^{1}$, Md Junayed Nayeem ${ }^{1}$ and Motohiko Sato ${ }^{1 *}$
}

\begin{abstract}
Glucose uptake and adenosine triphosphate (ATP) generation are important for the survival and growth of endothelial cells. An increase of glucose uptake under hypoxia was previously shown to be associated with the increased expression of glucose transporters (GLUTS). However, the regulation of GLUT trafficking to the cell surface has not been examined in detail. Here, we report the characterization of GLUT1 translocation to the plasma membrane during hypoxia in endothelial cells. Human umbilical vein endothelial cells (HUVECs) were exposed to hypoxia $\left(1 \% \mathrm{O}_{2}\right)$ for $12 \mathrm{~h}$, which significantly induced GLUT1 expression and translocation to the plasma membrane. GLUT1 translocation was associated with a decrease of intracellular ATP by hypoxia. Decreasing ATP levels with antimycin-A and 2-deoxyglucose induced GLUT1 translocation under normoxia. The induction of hypoxia-inducible factor-1a under normoxia did not influence the cell surface expression of GLUT1 or cellular ATP concentration. Interestingly, the translocation of GLUT1 induced by hypoxia was inhibited by the ATP-sensitive potassium (KATP) channel inhibitor glibenclamide, while the mitochondrial KATP channel inhibitor 5-HD did not influence GLUT1 translocation during hypoxia. These observations indicate that a decrease of intracellular ATP triggers GLUT1 translocation to the plasma membrane and is mediated by KATP channels, which would contribute to glucose uptake in HUVECs during hypoxia.
\end{abstract}

Keywords: Glucose transporters, Hypoxia, Endothelial cells, ATP, ATP-sensitive potassium channel

\section{Introduction}

Glucose is the essential source of energy for most cells. Glucose and other carbohydrates are transported from the surrounding fluid into cells by a family of membrane integral proteins called glucose transporters (GLUTs) [1]. To date, 14 GLUTs have been identified in humans, with GLUT1, GLUT3, and GLUT4 being the most commonly expressed in a wide variety of cells $[1,2]$. Physiological stimuli induce GLUT expression to facilitate the incorporation of an appropriate quantity of glucose for energy production. For example, hypoxia stimulates glucose transport into cells by increasing the expression of

*Correspondence: motosato@aichi-med-u.ac.jp

${ }^{1}$ Department of Physiology, Aichi Medical University, 1-1

Yazako-Karimata, Nagakute-City, Aichi 4801165, Japan

Full list of author information is available at the end of the article
GLUT1 [3, 4]. These processes are regulated to maintain homeostasis, and abnormal expression of GLUTs is often associated with human diseases [2]. The elevated expression of GLUT1 and/or GLUT3 is associated with poor survival in many solid cancers, including gastric cancer [5], breast cancer [6], lung cancer [7], and glioblastoma [8]. In addition, the cell surface localization of GLUTs is an important factor for glucose transport [2]. The insulin-mediated translocation of GLUT4 to the plasma membrane has been well characterized; however, the translocation mechanisms of other GLUTs have not been examined in detail.

Glucose uptake is critical for the growth of endothelial cells and angiogenesis, because energy production in endothelial cells is highly dependent on glycolysis [9, 10]. We previously demonstrated that the concentration of adenosine triphosphate (ATP) is a critical determinant 
of vascular endothelial growth factor receptor (VEGFR) signaling under hypoxia, which is important for angiogenesis [11]. Thus, under hypoxic conditions, the VEGFstimulated phosphorylation of VEGFR-2 is significantly inhibited, which is associated with a decrease of intracellular ATP, while the restoration of ATP levels recovers VEGFR-2 signaling. Furthermore, GLUT1 expression is increased by the exposure of endothelial cells to hypoxia [11]. However, the subcellular localization of GLUTs in endothelial cells, particularly under hypoxia, has not been examined in detail. This information is important for our understanding of angiogenic events when confronted with physiological challenges.

In this study, we examined the expression and intracellular localization of GLUT1 under hypoxia, particularly the trafficking of GLUT1 to the cell surface, which is important for glucose uptake by endothelial cells.

\section{Materials and methods Materials}

Human umbilical vein endothelial cells (HUVECs) were purchased from Lonza (Basel, Switzerland). A ReverTra Ace RT Kit was bought from TOYOBO (Osaka, Japan). Anti-GLUT1 (\#12939) and Alexa Fluor 488 anti-rabbit IgG antibodies were purchased from Cell Signaling Technology (Danvers, MA). Anti-GLUT3 (PA5-72331) was obtained from Thermo Fisher (Waltham, MA). $\beta$-actin antibody (AC-74), ATP-sensitive potassium (KATP) channel inhibitor glibenclamide, mitochondrial KATP channel inhibitor 5-HD, ATP inhibitor antimycin-A, 2-deoxyglucose (2-DG), and GLUT1 inhibitor BAY876 were acquired from Sigma-Aldrich (St. Louis, MO). The selectivity of BAY876 for GLUT1 is over 100 times greater than the $\mathrm{IC}_{50}$ for GLUT2, GLUT3, and GLUT4 [12]. An ATP Standard and ATP Assay Kit were obtained from TOYO B-Net (Tokyo, Japan). A Glucose Uptake Assay Kit was purchased from Cosmo Bio Co., Ltd. (\#MBR-PMG-K01E; Tokyo, Japan). Low-oxygen-containing gas $\left(1 \% \mathrm{O}_{2}, 5 \% \mathrm{CO}_{2}\right.$, and $\left.94 \% \mathrm{~N}_{2}\right)$ was obtained from Daiwa Shokai (Nagoya, Japan).

\section{Cell culture}

HUVECs were cultured in endothelial cell growth medium (EGM-2; Lonza, Basel, Switzerland) on fibronectin-coated culture dishes or plates (Corning, Schiphol, Netherlands). The cells were used between passages 3 and 7 for experiments. HUVECs were cultured in $6-, 12-$, or 96 -well plates to $70 \%-80 \%$ confluence. The cells were treated with glibenclamide $(10 \mu \mathrm{M})$ or 5-HD $(100 \mu \mathrm{M})$ for $1 \mathrm{~h}$ and exposed to hypoxia (1\% $\mathrm{O}_{2}, 5 \% \mathrm{CO}_{2}$ at $37^{\circ} \mathrm{C}$ ) for $12 \mathrm{~h}$ or cultured with antimycinA $(10 \mu \mathrm{M})$ or 2-DG $(1 \mathrm{mM})$ for $30 \mathrm{~min}$. HUVECs were also treated with $\mathrm{CoCl}_{2}(100 \mu \mathrm{M})$ and cultured for $3 \mathrm{~h}$ to mimic hypoxia. Hypoxia was maintained using a hypoxic chamber (AnaeroPack Rectangular Jar; MITSUBISHI GAS CHEMICAL COMPANY, Inc., Tokyo, Japan) with an oxygen monitor (OXY-1; ICHINEN JIKCO Co., Ltd., Tokyo, Japan).

\section{Real-time PCR}

Total RNA was extracted from HUVECs using an RNeasy Mini Kit (Qiagen, Hilden, Germany) according to the manufacturer's protocol and reverse transcribed to cDNA with the use of a ReverTra Ace RT Kit (TOYOBO). Realtime PCRs were run on a StepOnePlus system (Applied Biosystems, Foster City, CA). The following primers were used for PCR [11, 13]: GLUT1 forward, 5'-CGTCTTCAT CATCTTCAC TG-3'; GLUT1 reverse, 5'-CTCCTCGGG TGTCTTATC-3'; GLUT2 forward, 5'-CACACAAGA CCTGGAATTGACA-3'; GLUT2 reverse, 5'-CGGTCA TCCAGTGGAAGAC-3'; GLUT3 forward, 5'-CGGCTT CCTCATTAC CTTC-3'; GLUT3 reverse, 5'-GGCACG ACTTAGACATTGG-3'; GLUT4 forward, 5'-CTGGGC CTCACAGTGCTAC-3'; GLUT4 reverse, 5'-GTCAGG CGCTTCAGACTCTT-3'; GLUT5 forward, 5'-CATCAC TGTTGGCATCCTTGTG-3'; GLUT5 reverse, 5'-AGG ATCGGCCAGCCATCTAC-3'; Kir6.1 forward, 5'-CAA CTGCTGTGTCCAGAT-3'; Kir6.1 reverse, 5'-ATACGA ATGGTGATGTTGGA-3'; Kir6.2 forward, 5'-CATAGG CATTAGTGTAGT-3'; Kir6.2 reverse, 5'-TTATAGAAG AGGCAACTG- $3^{\prime}$; SUR1 forward, 5'-CAACTGCTG TGTCCAGAT-3'; SUR1 reverse, 5'-ATACGAATGGTG ATGTTGGA-3'; SUR2A forward, 5'-AAGCATTCG GTCATTGTAG-3'; SUR2A reverse, 5'-GCCACATAG TAGGTCTGA-3'; SUR2B forward, 5'-TGGAGAGGA TGTGGAGAA-3'; SUR2B reverse, 5'-CTGTAAGAA TGGTGAATGTGA T-3'; 18S rRNA forward, 5'-GTC TGTGATGCCCTTAGATG-3'; and 18S rRNA reverse, 5'-AGCTTATGACCCGCACTT AC-3'.

\section{Immunoblot analysis}

Total protein lysates were prepared by collecting experimental HUVECs in $1 \times$ sodium dodecyl sulfate (SDS)polyacrylamide gel electrophoresis (PAGE) sample buffer (65.8 mM Tris-HCl, pH 6.8, 2.1\% SDS, 26.3\% glycerol, $0.01 \%$ bromophenol blue) with protease inhibitor cocktail (Roche, Indianapolis, IN) and 4\% 2-mercaptoethanol. The protein lysates were homogenized with a $26 \mathrm{G}$ syringe needle and separated by SDS-PAGE. The separated proteins were transferred onto a PVDF membrane (Millipore, Billerica, MA). The membrane was blocked with Tris-buffered saline containing $5 \%$ bovine serum albumin (BSA; Sigma-Aldrich) and incubated with primary antibodies overnight at $4{ }^{\circ} \mathrm{C}$. The membrane was treated with horseradish peroxidase-conjugated secondary antibodies (GE Healthcare, Munich, Germany), and 
signals were detected using Immunostar LD western blotting detection reagents (Wako Pure Chemical Industries, Osaka, Japan) and a LAS 4000 image analyzer (GE Healthcare). For quantification, band intensity was analyzed with ImageJ software.

\section{Immunocytochemistry}

GLUT1 localization was determined by immunocytochemistry analysis based on a laboratory and antibody recommended protocol [14]. HUVECs were cultured on 13-mm poly-L-lysine-coated coverslips (Matsunami Glass, Osaka, Japan). The cells were washed with icecold phosphate-buffered saline (PBS), fixed with $4 \%$ paraformaldehyde in PBS for $10 \mathrm{~min}$, and incubated with $1 \% \mathrm{BSA}$ and $0.3 \mathrm{M}$ glycine in 0.1\% PBS-Tween for $1 \mathrm{~h}$ to permeabilize the cells and to block nonspecific protein-protein interactions. The cells were incubated with an anti-GLUT1 antibody (\#ab652, 1/200 dilution) overnight at $4{ }^{\circ} \mathrm{C}$. The cells were washed with PBS and incubated with Alexa Fluor 488 anti-rabbit IgG (green, 1/500 dilution) for $1 \mathrm{~h}$ in the dark. The cells were washed and incubated with DAPI (Life Technologies, Carlsbad, CA) in PBS for $5 \mathrm{~min}$. The cells were washed and mounted on slides with glass coverslips using Fluoro Keeper Antifade Reagent (Nacalai Tesque, Kyoto, Japan). Images were obtained with an LSM710 laser confocal microscope (Carl Zeiss, Jena, Germany).

\section{Flow cytometric analysis}

HUVECs were cultured in 6-well plates with EGM-2 to 70\%-80\% confluence. A FACS Canto II (BD Biosciences, Franklin Lakes, NJ) or BD LSRFortessa X-20 (BD Biosciences) was used to perform fluorescence-activated cell sorting (FACS). The cells were washed with ice-cold PBS, detached from the plates using Accutase (Innovative Cell Technologies, San Diego, CA), centrifuged, and washed with ice-cold PBS. The cells were fixed with $4 \%$ paraformaldehyde for $15 \mathrm{~min}$, washed with PBS, and labeled with a human anti-GLUT1 Alexa Fluor 700-conjugated antibody (FAB1418N, 1:100 dilution; R\&D Systems) or a human anti-GLUT1 Alexa Fluor 488-conjugated antibody (FAB1418G, 1:100 dilution; R\&D Systems) for $1 \mathrm{~h}$ on ice in the dark to detect cell surface GLUT1. Flow cytometric analysis and data acquisition were performed with the Canto II system or BD LSRFortessa X-20 system using FACS Diva software (BD Biosciences). FlowJo software (Tree Star, Ashland, OR) was used for data analysis.

\section{ATP analysis}

To analyze ATP levels in HUVECs, the cells were seeded in fibronectin-coated 96-well plates and cultured with EGM-2 overnight. For the ATP assay, the cells were treated with glibenclamide for $1 \mathrm{~h}$ and cultured under normoxia or exposed to hypoxia for $12 \mathrm{~h}$ or cultured with $\mathrm{CoCl}_{2}$ for $3 \mathrm{~h}$ or antimycin-A $(10 \mu \mathrm{M})$ or 2 -DG $(1 \mathrm{mM})$ for $30 \mathrm{~min}$ before measuring ATP concentration. Intracellular ATP concentration was measured using a luminescence-based ATP assay kit (TOYO B-Net) according to the manufacturer's instructions.

\section{2-DG uptake assay}

Glucose transport into HUVECs was measured using 2-DG with a Glucose Cellular Uptake Measurement Kit (Cosmo Bio Co., Ltd.) according to the manufacturer's instructions. HUVECs cultured in 12-well plates were washed with warm $\mathrm{KRPH}$ buffer $\left(1.2 \mathrm{mM} \mathrm{KH_{2 }} \mathrm{PO}_{4}\right.$, $1.2 \mathrm{mM} \mathrm{MgSO}_{4}, 1.3 \mathrm{mM} \mathrm{CaCl}_{2}, 118 \mathrm{mM} \mathrm{NaCl}, 5 \mathrm{mM}$ $\mathrm{KCl}, 30 \mathrm{mM}$ HEPES, $\mathrm{pH} 7.5)$ containing $2 \%$ BSA. The cells were incubated with $1 \mathrm{mM}$ of 2-DG in KRPH buffer for $20 \mathrm{~min}$ at $37^{\circ} \mathrm{C}$. Cellular 2-DG uptake was stopped by washing the cells with ice-cold PBS containing $200 \mu \mathrm{M}$ phloretin (Sigma-Aldrich). Cell lysates were collected in $1 \times$ sample buffer and heated at $80{ }^{\circ} \mathrm{C}$ for $15 \mathrm{~min}$. The lysates were centrifuged at $15,000 \times g$ for $20 \mathrm{~min}$ at $4{ }^{\circ} \mathrm{C}$ and the supernatants were collected. 2-DG uptake was measured using a fluorometric-based glucose uptake

\footnotetext{
(See figure on next page.)

Fig. 1 Glucose uptake and GLUT1 expression in HUVECs under normoxia and hypoxia. a Glucose uptake of HUVECs under normoxia and hypoxia. HUVECS were exposed to hypoxia, and glucose uptake was measured as described in the Materials and methods. Data are expressed as the mean \pm SEM from 6 independent experiments. ${ }^{*} P<0.05$ (Mann-Whitney test). $\mathbf{b}$ GLUT1 and GLUT3 expression in HUVECS under normoxia and hypoxia determined by immunoblotting. Data are expressed as the mean \pm SEM of 12 samples from 3 independent experiments. ${ }^{*} P<0.01$ vs. normoxia control of each condition (Mann-Whitney test). c Effect of the GLUT1 inhibitor BAY876 on glucose uptake in HUVECS. HUVECS were cultured under normoxia or hypoxia with BAY876 as described in the Materials and Methods. Data are expressed as a percentage of glucose uptake compared with vehicle control in each condition. Data are expressed as the mean \pm SEM from 5 independent results. ${ }^{*} P<0.01$ (one-way ANOVA). $\mathbf{d}$ Effect of GLUT1 inhibitor on sprouting of cells from choroid explants [16]. The explants were treated with vehicle or the GLUT1 inhibitor BAY876 for 4 days. Images were taken and the area of cell sprouting was quantified by ImageJ software. Data are the mean \pm SEM from 12 explants in 2 time-independent experiments with similar results. ${ }^{*} \mathrm{P}<0.01$ (one-way ANOVA with Dunnett's correction). e GLUT1 expression in HUVECS under normoxia and hypoxia determined by immunoblotting. Data are expressed as the mean \pm SEM from 6 independent experiments. Glb, glibenclamide $(10 \mu \mathrm{M}) .{ }^{*} P<0.01 \mathrm{vs}$. normoxia control of each condition (Mann-Whitney test)
} 

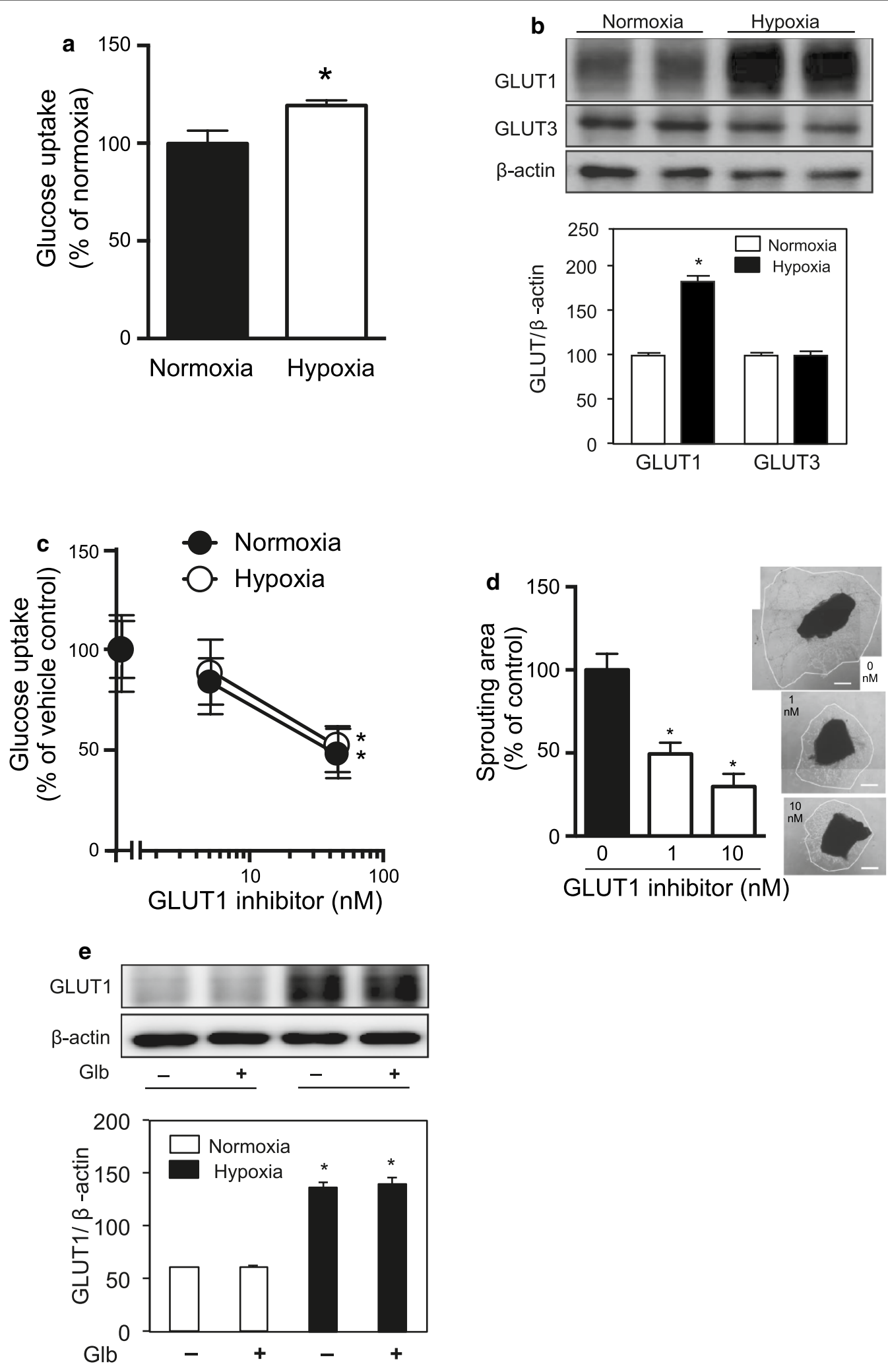
Table 1 Expression of GLUT1, GLUT3, and KATP channel subunits in HUVECs under normoxia and hypoxia

\begin{tabular}{llll}
\hline Gene name & $\begin{array}{l}\text { Normoxia } \\
\text { Mean } \pm \text { SEM }\end{array}$ & $\begin{array}{l}\text { Hypoxia } \\
\text { Mean } \pm \text { SEM }\end{array}$ & P Value \\
\hline GLUT1 & $0.483 \pm 0.041$ & $1.729 \pm 0.149$ & $<0.01$ \\
GLUT3 & $0.708 \pm 0.172$ & $1.544 \pm 0.148$ & $<0.05$ \\
Kir6.1 & $0.868 \pm 0.080$ & $1.044 \pm 0.126$ & n.s. \\
Kir6.2 & $1.223 \pm 0.823$ & $0.844 \pm 0.229$ & n.s. \\
SUR-1 & $0.997 \pm 0.317$ & $1.083 \pm 0.122$ & n.s. \\
SUR-2A & $1.117 \pm 0.216$ & $1.035 \pm 0.130$ & n.s. \\
SUR-2B & $0.899 \pm 0.110$ & $0.972 \pm 0.039$ & n.s. \\
\hline
\end{tabular}

The mRNA levels of GLUTs and KATP channel subunits were analyzed by realtime PCR $[11,13]$. Data are shown as relative values normalized with 18S RNA. Data are expressed as the mean \pm SEM of 4 independent experiments. $P$-values were analyzed by an unpaired $t$-test. n.s. not significant

assay kit (Cosmo Bio Co., Ltd.) according to the manufacturer's instructions.

\section{Miscellaneous procedures and data analysis}

Further details on the miscellaneous procedures are provided elsewhere $[15,16]$. Data are expressed as the mean \pm standard error of the mean (SEM) from independent experiments. Statistical analysis was performed using an unpaired $t$ test, Mann-Whitney test, or oneway analysis of variance (ANOVA) with post hoc analysis. Significant differences between the control and test groups were evaluated with P-values less than 0.05 and 0.01 . All statistical analyses were performed with IBM SPSS software (Armonk, NY) [17].

\section{Results}

\section{Hypoxia induces glucose uptake in HUVECs}

We first examined the influence of hypoxia on glucose uptake and GLUT expression in HUVECs. Glucose uptake was increased by $19 \%$ under hypoxia $\left(1 \% \mathrm{O}_{2}, 12 \mathrm{~h}\right)$ $(19.3 \pm 2.6$ vs. normoxia, mean \pm SEM, $p<0.05)$ (Fig. 1a), which was associated with an increase in GLUT1 protein expression (Fig. 1b). At the mRNA level, an increase of GLUT1 and GLUT3 expression was detected; however, there was no increase in the expression of GLUT3 protein. The expression of GLUT2, GLUT4, and GLUT5 mRNA was not detected in HUVECs, as previously reported (Table 1) [18]. Glucose uptake was significantly reduced by the GLUT1 inhibitor BAY876 under normoxia and hypoxia (Fig. 1c). These data indicated the isoform-specific regulation of GLUTs and the importance of GLUT1 for glucose uptake in vascular endothelial cells under hypoxia.

The role of GLUT1 was examined further using a sprouting assay of mouse choroid, which is an ex vivo model of angiogenesis (Fig. 1d) [16]. Cultured choroid fragments in the Matrigel (BD Biosciences) initiated the outgrowth of endothelial cells by VEGF [16]. The GLUT1 inhibitor BAY876 suppressed the outgrowth of cells from the explants, suggesting the importance of GLUT1 for the growth of endothelial cells and angiogenesis.

\section{Hypoxia induces GLUT1 translocation to the plasma membrane}

In addition to the cellular expression of GLUT1, its cell surface localization is another critical factor influencing glucose uptake [2]. The cell surface localization of GLUT1 was analyzed by FACS using a fluorescent dyeconjugated anti-GLUT1 antibody without permeabilization of the cells. The GLUT1 signal on the cell surface was significantly increased by exposure of HUVECs to hypoxia (Fig. 2a). The cell surface localization of GLUT1 was confirmed by fluorescent immunostaining in HUVECs (Fig. 3). The GLUT1 signal was increased at the cell surface under hypoxia (Fig. 3a, b). These data suggest that hypoxia triggers the translocation of GLUT1 to the cell surface. Interestingly, GLUT1 was increased at the cell surface, even after 3-h exposure of the cells to hypoxia (Fig. 4a), and was markedly decreased by reoxygenation (Fig. 4b), suggesting the dynamic regulation of cell-surface GLUT1 in response to the oxygen levels of cells.

\section{Decrease of ATP triggers GLUT1 translocation to the plasma membrane}

Cellular ATP was decreased by exposing the cells to hypoxia as reported previously (Fig. 5a) [11], which may be associated with GLUT1 translocation. The cell surface expression of GLUT1 was determined in the presence of antimycin-A or 2-DG, because they decrease cellular ATP even under normoxia (Fig. 5b) [11]. Interestingly, FACS analysis indicated that antimycin-A and 2-DG induced GLUT1 translocation to the plasma membrane (Fig. 6). This was also confirmed by immunofluorescence staining of GLUT1 (Fig. 7), suggesting that a decrease of ATP was key for GLUT1 translocation.

\section{KATP channels are involved in hypoxia-induced GLUT1 translocation}

To identify which molecule triggers the translocation of GLUT1 associated with a reduction of ATP, we examined KATP channels, which open following a decrease of intracellular ATP. HUVECs expressed KATP channel subunits, but their levels were not changed significantly during hypoxia (Table 1). Next, we analyzed the effect of glibenclamide, which blocks KATP channels, on GLUT1 translocation by FACS analysis and immunofluorescence staining (Figs. 2a and 3). Interestingly, glibenclamide 

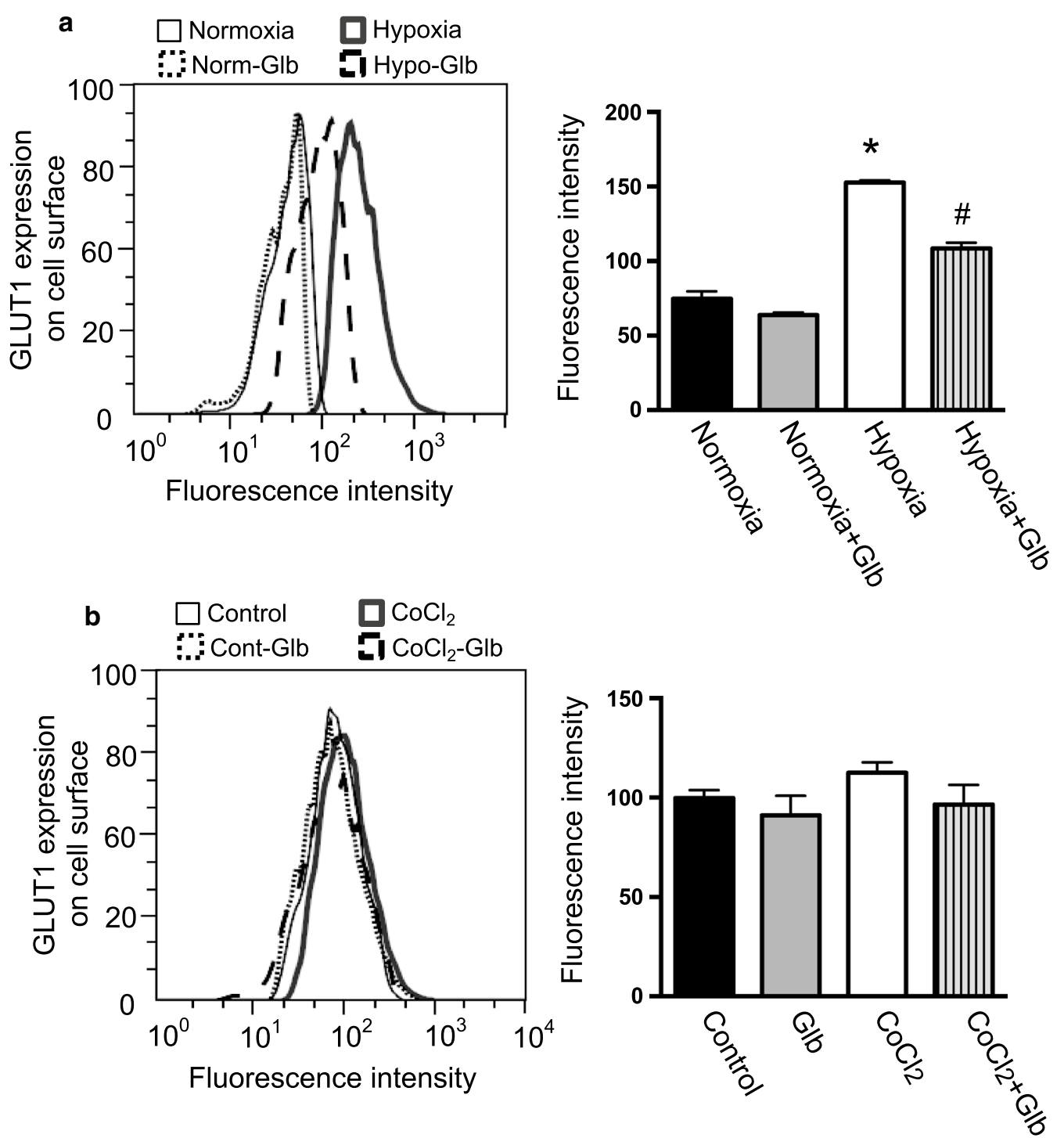

Fig. 2 Effect of hypoxia or reagents on the cell surface expression of GLUT1. a HUVECs were cultured under normoxia or hypoxia as described in the Materials and Methods. In some experiments, the cells were treated with glibenclamide (Glb, $10 \mu \mathrm{M})$. The cells were labeled a human anti-GLUT1 Alexa Fluor 700-conjugated antibody to detect cell surface GLUT1. b Effect of $\mathrm{CoCl}_{2}$ and/or glibenclamide on the cell surface expression of GLUT1. HUVECs were treated with $\mathrm{CoCl}_{2}(100 \mu \mathrm{M})$ and/or glibenclamide $(\mathrm{Glb}, 10 \mu \mathrm{M})$. GLUT1 at the cell surface was determined as described in $(\mathbf{a}) .(\mathbf{a}, \mathbf{b})$ The histograms represent cell counts (Y-axis, linear scale) versus fluorescence intensity (X-axis, log scale). Fluorescence mean intensity obtained from the histograms was quantified and shown in bar graphs (right panels). Data are the mean \pm SEM from 4 independent experiments. ${ }^{*} P<0.01$ versus normoxia control (Mann-Whitney test). ${ }^{\#} P<0.01$ versus hypoxia without glibenclamide treatment (Mann-Whitney test)

inhibited the hypoxia-induced translocation of GLUT1 to the cell surface in both analyses (Figs. 2a and 3). Furthermore, glibenclamide also inhibited the translocation of GLUT1 triggered by a decrease of ATP following treatment with antimycin-A and 2-DG under normoxia (Figs. 6 and 7). Treatment with glibenclamide reportedly reduces the expression of GLUT1 in human chondrocytes [13]; however, in HUVECs, it did not influence GLUT1 expression or intracellular ATP concentration
(Figs. 1e and 5a-d). These data indicated that KATP channels are involved in the hypoxia-induced translocation of GLUT1 by sensing a decrease of ATP levels.

\section{Mitochondrial KATP channels are not involved in GLUT1 translocation}

Glibenclamide blocks KATP channels in the plasma membrane and other organelles including mitochondria $[19,20]$. Therefore, we examined the role of 


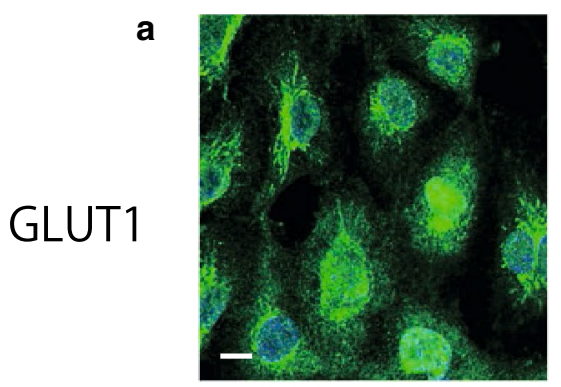

Normoxia

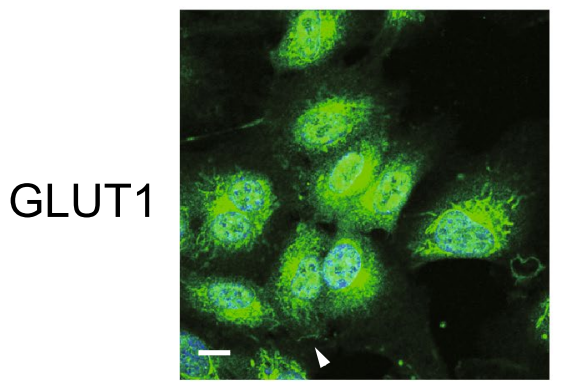

Normoxia+Glb

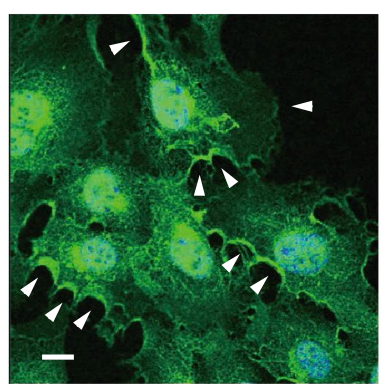

Hypoxia



Hypoxia+Glb

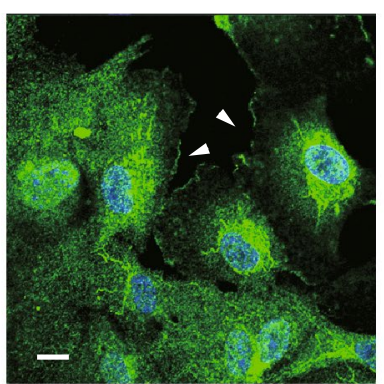

$\mathrm{CoCl} 2$

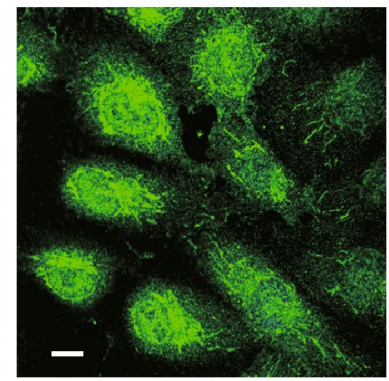

$\mathrm{CoCl}_{2}+\mathrm{Glb}$

b

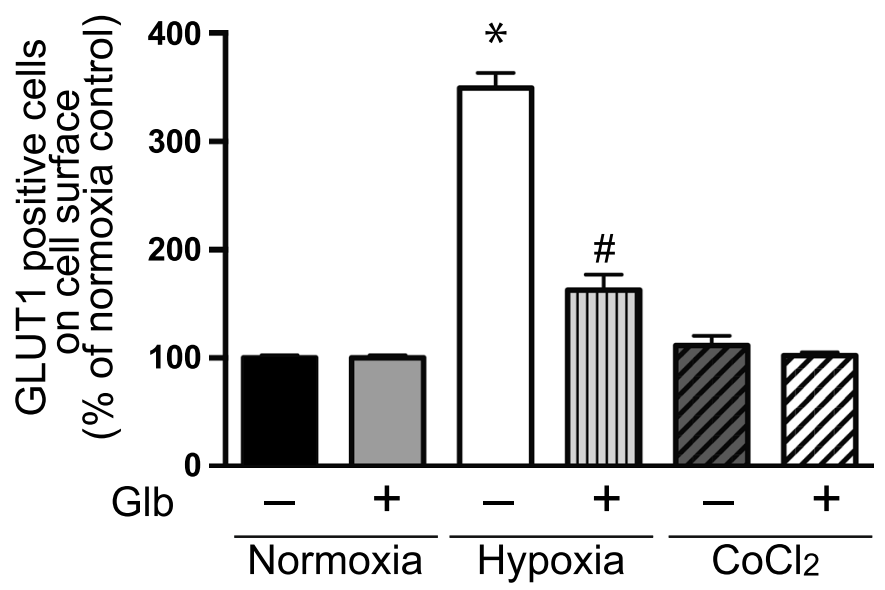

Fig. 3 GLUT1 expression on the cell surface. a HUVECs were cultured under normoxia or hypoxia as described in the Materials and Methods. In some experiments, the cells were treated with glibenclamide (Glb, $10 \mu \mathrm{M})$. a The cellular distribution of GLUT1 was determined by immunofluorescence staining. HUVECs were fixed with $4 \%$ paraformaldehyde and stained with an anti-GLUT1 antibody (green). Images were obtained with a confocal fluorescence microscope as described in the Materials and Methods. Representative images are shown from 4 independent experiments with similar results. Scale bars, $25 \mu \mathrm{m}$. b The number of cells in which GLUT1 was observed at the cell membrane was counted in approximately 100 cells from 4 or 5 independent experiments. Data are the mean \pm SEM from 4 independent experiments. ${ }^{*} P<0.01$ versus normoxia control (unpaired $t$-test). ${ }^{\#} P<0.01$ versus hypoxia without glibenclamide treatment (unpaired $t$-test)

mitochondrial KATP channels on the hypoxia-induced translocation of GLUT1. Interestingly, the mitochondrial KATP channel blocker 5-HD did not influence hypoxiainduced GLUT1 translocation, as determined by FACS analysis and immunofluorescence staining (Fig. 8). These data indicated that KATP channels in the plasma membrane or other organelles, but not in mitochondria, are involved in the hypoxia-induced translocation of GLUT1. 



Fig. 4 Effect of hypoxia and reoxygenation on the cell-surface expression of GLUT1. a HUVECs were cultured under normoxia or $3 \mathrm{~h}$ or $12 \mathrm{~h}$ hypoxia, as described in the Materials and Methods. The cells were labeled with a human anti-GLUT1 Alexa Fluor ${ }^{\circledR} 488$-conjugated antibody to detect cell-surface GLUT1. $\mathbf{b}$ Effect of re-oxygenation on the cell-surface expression of GLUT1. HUVECs were cultured under $12 \mathrm{~h}$ hypoxia or $12 \mathrm{~h}$ normoxia following $12 \mathrm{~h}$ hypoxia. GLUT1 at the cell surface was determined as described in $\mathbf{a}$. (a, b) The histograms represent cell counts (Y-axis, linear scale) versus fluorescence intensity (X-axis, log scale). Fluorescence mean intensity obtained from the histograms was quantified and shown in bar graphs (right panels). Data are the mean \pm SEM from 4 independent experiments. ${ }^{*} P<0.01,{ }^{\#} P<0.05$ vs. normoxia control (Mann-Whitney test). ${ }^{\$} P<0.01$ vs. hypoxia (12 h) (Mann-Whitney test)

\section{HIF-1a does not induce GLUT1 translocation}

A number of genes that are induced under hypoxia may also have a role in GLUT1 translocation. Hypoxia inducible factor-1 (HIF-1) is an important regulator of the metabolic adaptation of cells to hypoxia. $\mathrm{CoCl}_{2}$ stabilizes HIF- $1 \alpha$ and mimics the hypoxic environment in cells [21]. $\mathrm{CoCl}_{2}$ increased GLUT1 expression (Fig. 5d) [11]; however, it did not influence intracellular 
ATP concentration (Fig. 5c) or GLUT1 translocation, as determined by FACS analysis and immunofluorescence staining (Figs. $2 \mathrm{~b}$ and 3 ). These data indicated that the increased cellular expression of HIF- $1 \alpha$ did not induce a change in the level of GLUT1 in the plasma membrane, but another signal was required to trigger GLUT1 translocation to the plasma membrane.

\section{Discussion}

In this study, we demonstrated that the exposure of endothelial cells to hypoxia induced the translocation of GLUT1 to the cell surface. GLUT1 translocation was associated with a decrease of intracellular ATP, but not an increase of HIF-1. Further analysis suggested that KATP channels were involved in GLUT1 translocation. The ATP-triggered translocation of GLUT1 to the plasma membrane may be a part of a feedback mechanism between metabolic status and glucose uptake, which could contribute to glucose uptake during hypoxia and the angiogenic events of endothelial cells.

Glucose uptake and glycolysis are critical processes for energy production in endothelial cells. Glucose uptake was mediated by GLUT1 and GLUT3 in HUVECs (Table 1) [18]. GLUT3 is predominantly expressed in neurons and may play a role in angiogenesis in glioblastoma [22]. GLUT1 is the most important glucose transporter for a variety of cells [3, 4, 23]. In HUVECs, the GLUT1 inhibitor BAY876 significantly reduced glucose uptake and suppressed the outgrowth of endothelial cells from explants (Fig. 1), suggesting the importance of GLUT1 for angiogenic events in endothelial cells. Under hypoxia, GLUT1 expression was increased to facilitate glucose uptake. In addition to the levels of GLUTs, their cell surface localization is another determinant of glucose uptake [2]. In a series of experiments, the increase of cellular GLUT1 expression induced by HIF-1 was not concomitant with an increase of GLUT1 in the plasma membrane (Figs. 2b, 3, and $5 \mathrm{~d}$ ), suggesting that another signal was required to trigger GLUT1 translocation.
However, GLUT1 trafficking in endothelial cells has not been characterized in detail.

Hypoxia significantly increased the cell surface localization of GLUT1 in HUVECs. The translocation of GLUT4 has been characterized in detail, in which its translocation is induced by the activation of insulin receptors and the subsequent activation of the phosphatidylinositol 3-kinase (PI3K) and AKT pathway [2]. Several factors are reported to induce the translocation of GLUT1 to the plasma membrane, including interleukin 3 (IL-3) [24], nitric oxide [25], low-density lipoprotein (LDL) [26], insulin [27], dehydroepiandrosterone (DEHA) [28], ischemia [27], and the combination of hypoxia and high glucose concentration [29]. PI3K inhibition [28] and AKT knockdown [30] block the translocation of GLUT1 induced by insulin [28, 30] and DEHA [28], as observed for GLUT4. Similarly, PI3K inhibition also attenuates the translocation of GLUT1 induced by IL-3 [24], LDL, and the combination of hypoxia with high glucose concentration [29]. However, PI3K inhibition fails to block the translocation of GLUT1 induced by ischemia, suggesting the existence of another pathway regulating GLUT1 trafficking [27]. In this study, hypoxia clearly induced GLUT1 translocation in HUVECs, which was associated with a decrease of ATP concentration. A decrease of intracellular ATP was also related to the translocation of GLUT1 to the plasma membrane in a malaria-infected hepatocyte cell line [31], suggesting the existence of a mechanism triggered by an ATP-sensing molecule leading to GLUT1 translocation.

KATP channels are potential ATP-sensing molecules [32]. KATP channels consist of a large macromolecular complex in which four inwardly rectifying potassium channel (Kir6.x) subunits form a central pore surrounded by four regulatory sulphonyl urea receptor (SUR) subunits [33, 34]. Changes in intracellular ATP concentrations regulate KATP channel activity; higher ATP concentrations in cells inhibit or close KATP channels by binding to Kir6.x, while Mg-nucleotide binding/hydrolysis at the nucleotide-binding domains

\footnotetext{
(See figure on next page.)

Fig. 5 ATP concentration and GLUT1 expression in HUVECs. a Intracellular ATP production was measured following culture of the cells under normoxia or hypoxia, with or without glibenclamide $(\mathrm{Glb}, 10 \mu \mathrm{M})$, as described in the Materials and Methods. Data are expressed as the mean \pm SEM from 4 independent experiments. ${ }^{*} P<0.01$ vs. normoxic control without glibenclamide treatment (Mann-Whitney test). $\mathbf{b}$ HUVECs in 96 -well plates were treated with antimycin-A (Anti-A, $10 \mu \mathrm{M}$ ) or 2-deoxyglucose (2-DG, $1 \mathrm{mM}$ ) for $30 \mathrm{~min}$ at normoxia. In some groups, the cells were also treated with glibenclamide $(\mathrm{Glb}, 10 \mu \mathrm{M})$. ATP was measured as described in the Materials and Methods. Data are from experiments performed in duplicate. ${ }^{*} P<0.01$ compared with control without glibenclamide treatment (Mann-Whitney test). c HUVECs were treated with $\mathrm{CoCl}_{2}(100 \mu \mathrm{M})$ with or without glibenclamide (Glb, $10 \mu \mathrm{M})$. Intracellular ATP concentration was analyzed as described in the "Materials and methods". Data are expressed as the mean \pm SEM from 4 independent experiments. No statistical significance was detected by the Mann-Whitney test. d GLUT1 expression determined by immunoblotting following treatment with antimycin-A (Anti-A, $10 \mu \mathrm{M})$, 2-deoxyglucose (2-DG, $1 \mathrm{mM}), \mathrm{CoCl}_{2}(100 \mu \mathrm{M})$, and/or glibenclamide $(\mathrm{Glb}, 10 \mu \mathrm{M})$. Data are expressed as the mean \pm SEM from 4 independent experiments. $\# P<0.05$ versus vehicle control without glibenclamide treatment (Mann-Whitney test)
} 

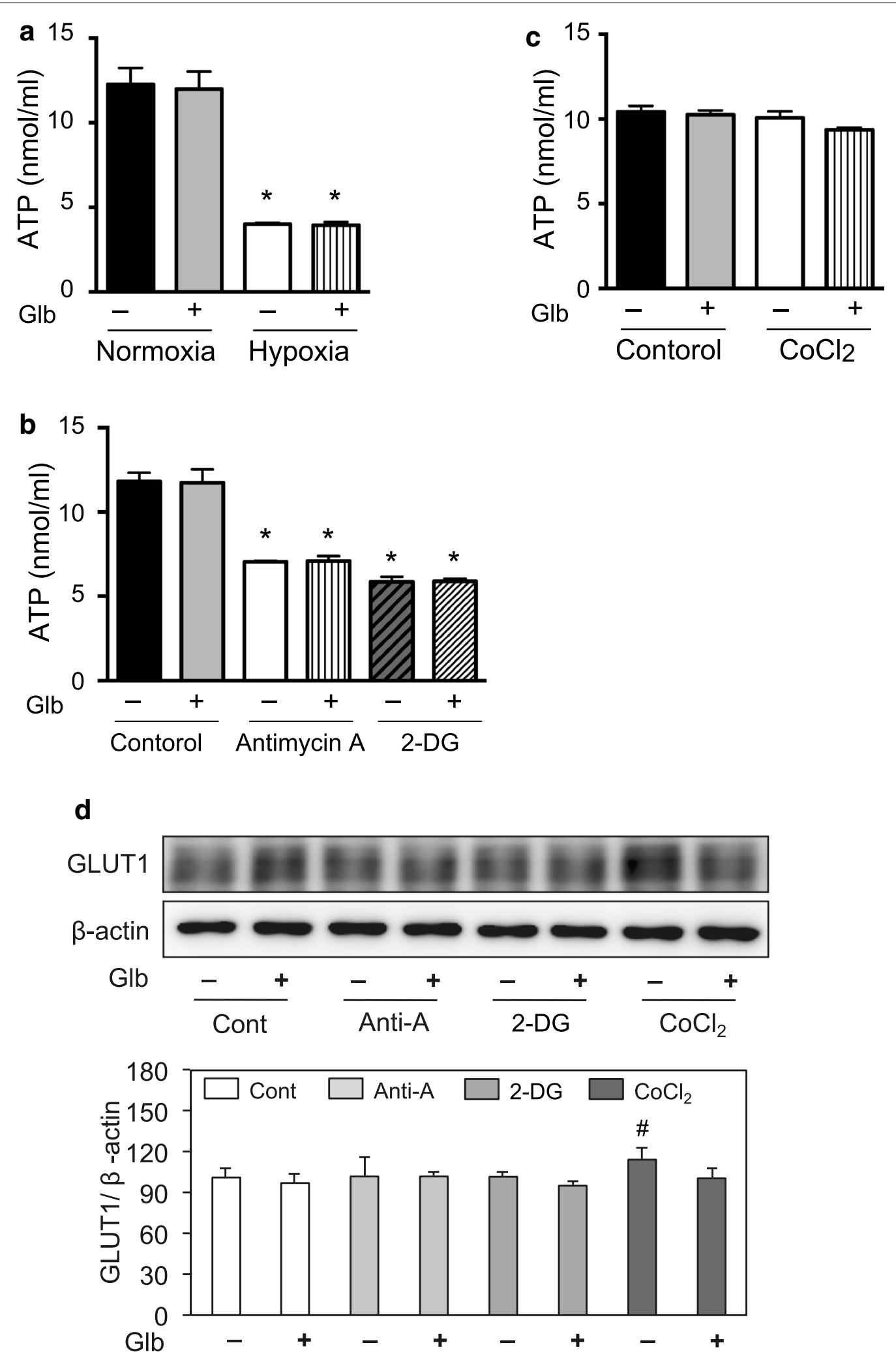

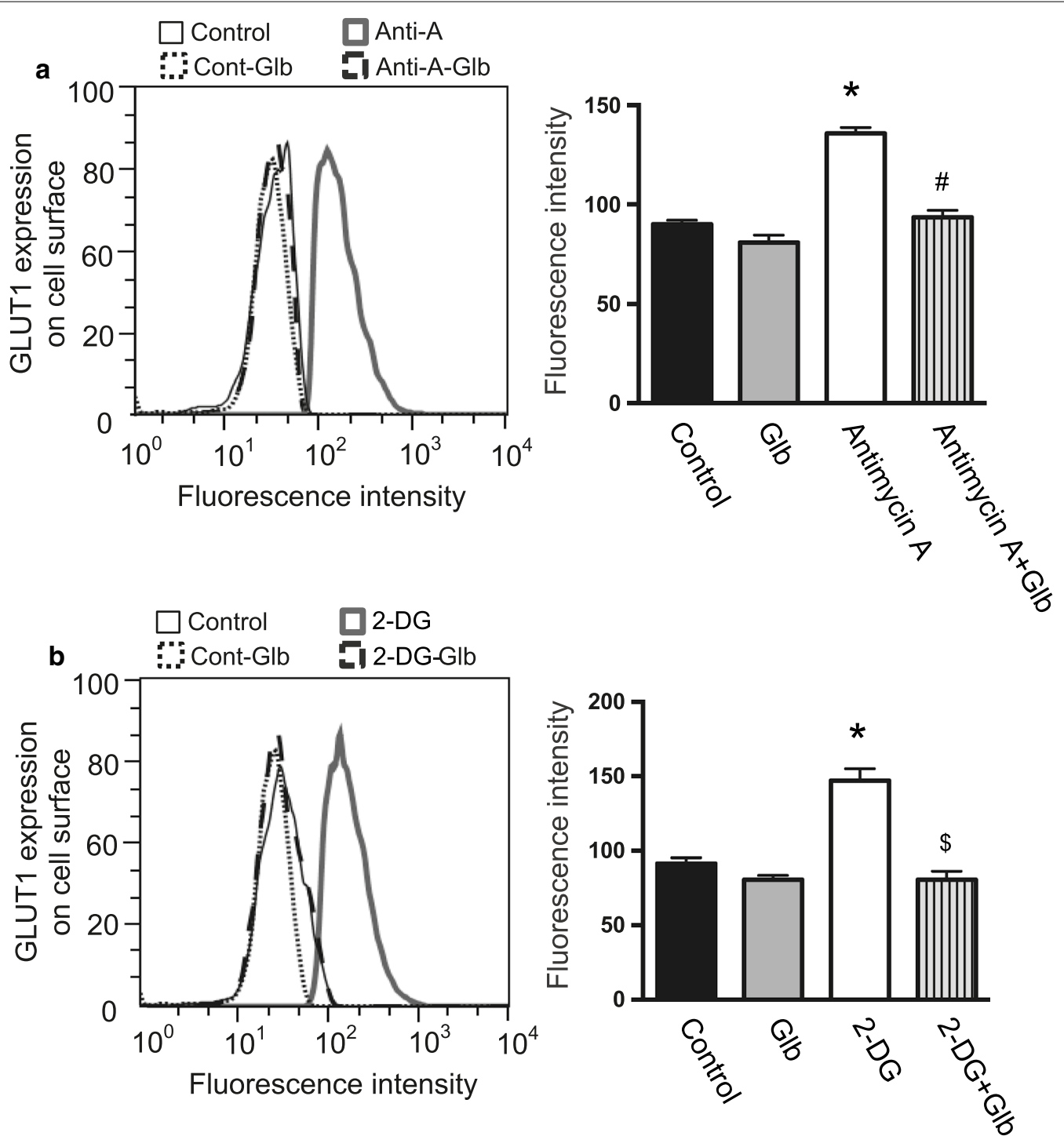

Fig. 6 Effects of reagents decreasing cellular ATP levels on the cell surface expression of GLUT1. HUVECs were treated with antimycin-A (Anti-A, $10 \mu \mathrm{M})(\mathbf{a})$ or 2-deoxyglucose (2-DG, $1 \mathrm{mM}$ ) (b) for 30 min at normoxia. In some groups, the cells were also treated with glibenclamide (Glb, $10 \mu \mathrm{MM})$. The cells were labeled with a human anti-GLUT1 Alexa Fluor 700-conjugated antibody to detect GLUT1 on the cell surface. (a, b) The histograms represent cell counts (Y-axis, linear scale) versus fluorescence intensity (X-axis, log scale). Fluorescence mean intensity obtained from the histograms was quantified and shown in bar graphs (right panels). Data are the mean \pm SEM from 4 independent experiments. ${ }^{*} P<0.01$ versus normoxia control (Mann-Whitney test). ${ }^{\#} P<0.01$ versus antimycin-A (Mann-Whitney test). ${ }^{\$} P<0.01$ versus 2 -deoxyglucose (Mann-Whitney test)

of SUR stimulates channel opening [35]. The balance between these stimulatory and inhibitory effects determines the level of channel activity. HUVECs expressed the necessary components to assemble functional KATP channels, and their levels were not changed under hypoxia compared with normoxia (Table 1). Glibenclamide, which binds to SUR subunits and inhibits KATP channel activity in the plasma membrane and other organelles including mitochondria, inhibited the hypoxia-induced translocation of GLUT1. However, 5-HD, a mitochondrial KATP channel inhibitor, had no effect on GLUT1 translocation during hypoxia. These data suggested that KATP channels in the plasma membrane or other organelles rather than mitochondria are involved in the hypoxia-induced translocation of GLUT1. The opening of KATP channels in the plasma membrane, stimulated by low levels of intracellular ATP, typically decreases the membrane potential of endothelial cells. Currently, the mechanism by which hyperpolarization induces the translocation of molecules is not well-defined and thus further studies are required. In addition to KATP channels in the 


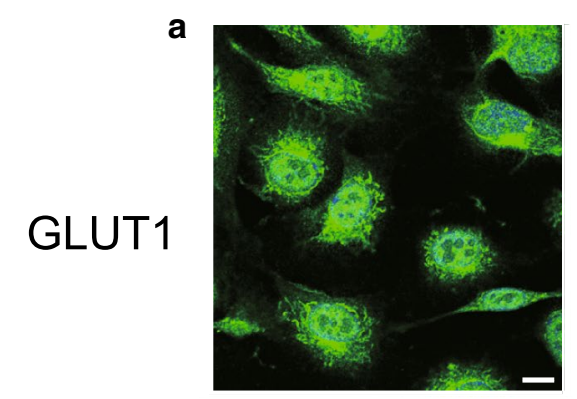

Control

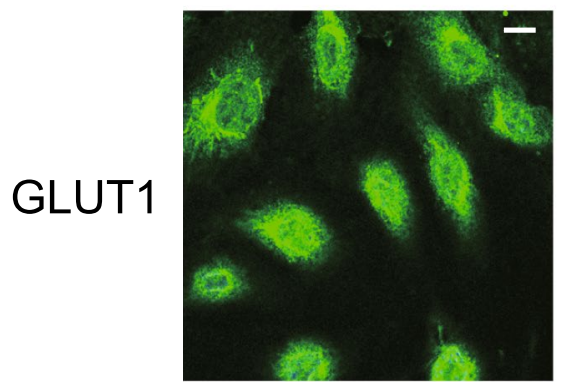

Glb

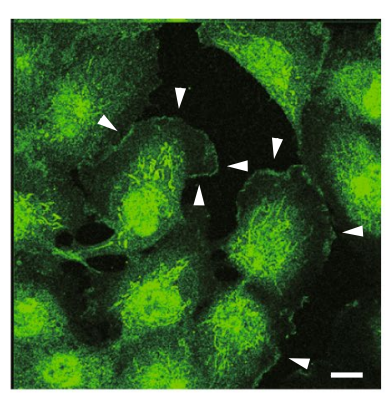

Antimycin A



Antimycin A + Glb

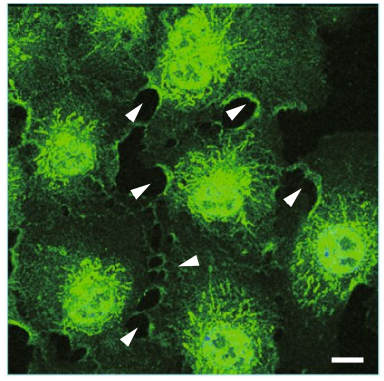

2-DG

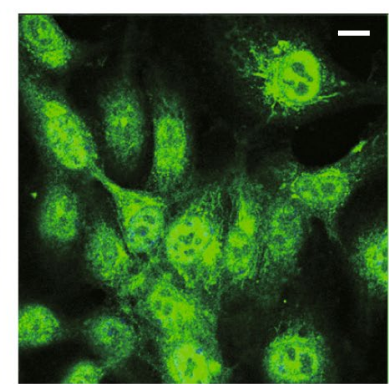

$2-D G+G l b$

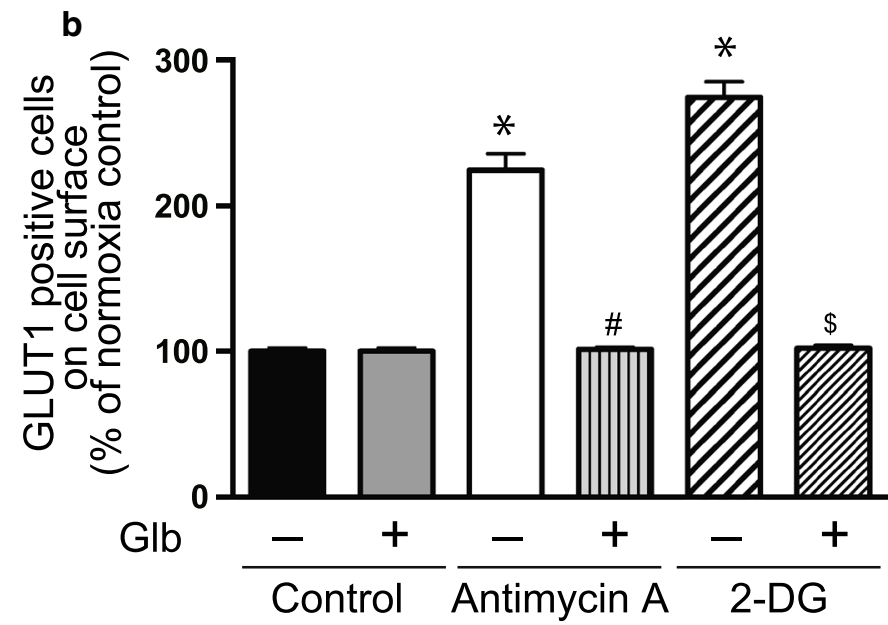

Fig. 7 Effects of reagents decreasing cellular ATP levels on the subcellular localization of GLUT1. a HUVECs were treated with antimycin-A (Anti-A, $10 \mu \mathrm{M}$ ) or 2-deoxyglucose (2-DG, $1 \mathrm{mM}$ ) with or without glibenclamide (Glb, $10 \mu \mathrm{M})$ under normoxia. The cellular distribution of GLUT1 was determined by immunofluorescence staining as described in the Materials and Methods. HUVECs were stained with an anti-GLUT1 antibody (green). Images were obtained with a confocal fluorescence microscope as described in the "Materials and methods". Representative images are shown from 4 independent experiments with similar results. Scale bars, $25 \mu \mathrm{m}$. b The number of cells in which GLUT1 was observed at the cell membrane was counted in approximately 100 cells from 4 or 5 independent experiments. Data are the mean \pm SEM from 4 independent experiments. ${ }^{*} P<0.01$ versus normoxia control without glibenclamide treatment (unpaired $t$-test). ${ }^{\#} P<0.01$ versus antimycin-A without glibenclamide treatment (unpaired $t$-test). ${ }^{\$} P<0.01$ versus 2 -deoxyglucose without glibenclamide treatment (unpaired $t$-test)

plasma membrane, the presence of KATP channels in the endoplasmic reticulum and nuclear membrane has also been reported [19, 20, 36]. KATP channels in such organelles may be involved in the translocation of GLUT1 under hypoxia.

\section{Conclusion}

In conclusion, we have demonstrated the isoform-specific expression of GLUTs and the translocation of GLUT1 in vascular endothelial cells under hypoxia. GLUT1 translocation was induced by a decrease of intracellular ATP 



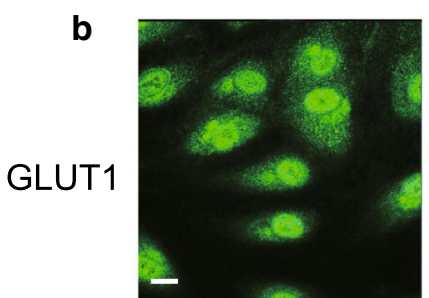

Normoxia

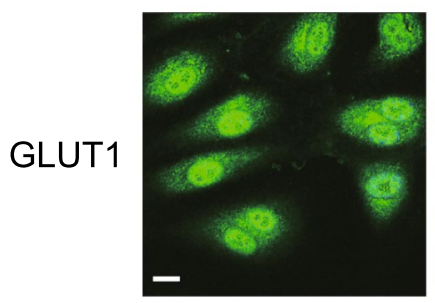

Normoxia + 5HD

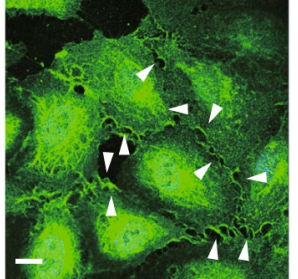

Hypoxia

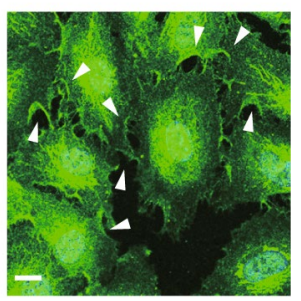

Hypoxia + 5HD

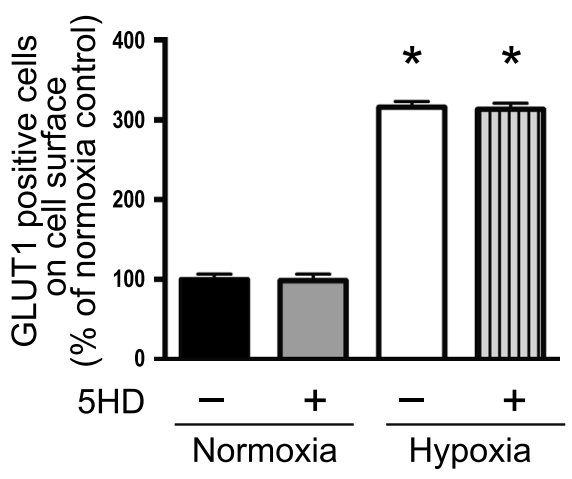

Fig. 8 Effects of the mitochondrial KATP channel inhibitor 5-HD on the cell surface expression of GLUT1. HUVECs were cultured under normoxia or hypoxia with or without 5-HD (100 MM). a Cell surface expression of GLUT1 determined by flow cytometric analysis. The cells were labeled with a human anti-GLUT1 Alexa Fluor 700-conjugated antibody to detect cell surface GLUT1. Left panel: The histograms represent cell counts (Y-axis, linear scale) versus fluorescence intensity (X-axis, log scale). Right panel: Fluorescence mean intensity obtained from the histograms was quantified and shown in bar graphs (right panels). Data are the mean \pm SEM from 4 independent experiments. ${ }^{*} P<0.01$ versus normoxia control (Mann-Whitney test). $\mathbf{b}$ The cellular distribution of GLUT1 was determined by immunofluorescence staining as described in the Materials and Methods. HUVECS were stained with an anti-GLUT1 antibody (green). Left panel: Images were obtained with a confocal fluorescence microscope as described in the "Materials and methods". Representative images are shown from 4 independent experiments with similar results. Scale bars, $25 \mu \mathrm{m}$. Right panel: The number of cells in which GLUT1 was observed at the cell membrane was counted in approximately 100 cells from 4 independent experiments. Data are the mean \pm SEM from 4 independent experiments. ${ }^{*} P<0.01$ versus normoxia control (Mann-Whitney test)

and was mediated by KATP channels. Glucose uptake and subsequent ATP production are important for angiogenic events in endothelial cells. Our data indicate the existence of functional feedback between metabolic status and GLUT1 translocation, which will contribute to a more precise understanding of the induction of angiogenesis by hypoxia and tumors.

\section{Acknowledgements}

We thank Ms. Rie Takahashi for technical assistance.

\section{Authors' contributions}

MS conceived and coordinated the study and wrote the paper. AAM designed and performed the study and wrote the paper. $\mathrm{HH}, \mathrm{AY}$, and MJN provided substantial technical assistance and contributed to the preparation of the figures. All authors analyzed the results and approved the final version of the manuscript. All authors read and approved the final manuscript. 


\section{Funding}

This study was supported by JSPS KAKENHI Grant Number JP19K07309 (to M.S.); Nittoku Asia Foreign Student Scholarship (to A.A.M.); Three Es Foundation Scholarship (to MJN).

\section{Availability of data and materials}

All relevant data are within the paper.

\section{Ethics approval and consent to participate} Not applicable.

\section{Consent for publication}

Not applicable.

\section{Competing interests}

The authors declare that they have no conflicts of interest with the contents of this article.

\section{Author details}

${ }^{1}$ Department of Physiology, Aichi Medical University, 1-1 Yazako-Karimata, Nagakute-City, Aichi 4801165, Japan. ${ }^{2}$ Department of Biochemistry and Molecular Biology, Mawlana Bhashani Science and Technology University, Santosh, Tangail 1902, Bangladesh.

Received: 29 January 2020 Accepted: 11 September 2020

Published online: 22 September 2020

\section{References}

1. Jia B, Yuan P, Lan WJ, Xuan YH, Jeon CO (2019) New insight into the classification and evolution of glucose transporters in the Metazoa. FASEB J 33:7519-7528

2. Ancey PB, Contat C, Meylan E (2018) Glucose transporters in cancer-from tumor cells to the tumor microenvironment. FEBS J 285:2926-2943

3. Wood IS, Wang B, Lorente-Cebrian S, Trayhurn P (2007) Hypoxia increases expression of selective facilitative glucose transporters (GLUT) and 2-deoxy-D-glucose uptake in human adipocytes. Biochem Biophys Res Commun 361:468-473

4. Zhang JZ, Behrooz A, Ismail-Beigi F (1999) Regulation of glucose transport by hypoxia. Am J Kidney Dis 34:189-202

5. Berlth F, Monig S, Pinther B, Grimminger P, Maus M, Schlosser H, Plum P, Warnecke-Eberz U, Harismendy O, Drebber U, Bollschweiler E, Holscher A, Alakus H (2015) Both GLUT-1 and GLUT-14 are independent prognostic factors in gastric adenocarcinoma. Ann Surg Oncol 22(Suppl 3):S822-\$831

6. Wang J, Ye C, Chen C, Xiong H, Xie B, Zhou J, Chen Y, Zheng S, Wang L (2017) Glucose transporter GLUT1 expression and clinical outcome in solid tumors: a systematic review and meta-analysis. Oncotarget 8:16875-16886

7. Osugi J, Yamaura T, Muto S, Okabe N, Matsumura Y, Hoshino M, Higuchi M, Suzuki H, Gotoh M (2015) Prognostic impact of the combination of glucose transporter 1 and ATP citrate lyase in node-negative patients with non-small lung cancer. Lung Cancer 88:310-318

8. Flavahan WA, Wu Q, Hitomi M, Rahim N, Kim Y, Sloan AE, Weil RJ, Nakano I, Sarkaria JN, Stringer BW, Day BW, Li M, Lathia JD, Rich JN, Hjelmeland AB (2013) Brain tumor initiating cells adapt to restricted nutrition through preferential glucose uptake. Nat Neurosci 16:1373-1382

9. De Bock K, Georgiadou M, Schoors S, Kuchnio A, Wong BW, Cantelmo AR, Quaegebeur A, Ghesquiere B, Cauwenberghs S, Eelen G, Phng LK, Betz I, Tembuyser B, Brepoels K, Welti J, Geudens I, Segura I, Cruys B, Bifari F, Decimo I, Blanco R, Wyns S, Vangindertael J, Rocha S, Collins RT, Munck S, Daelemans D, Imamura H, Devlieger R, Rider M, Van Veldhoven PP, Schuit F, Bartrons R, Hofkens J, Fraisl P, Telang S, Deberardinis RJ, Schoonjans L, Vinckier S, Chesney J, Gerhardt H, Dewerchin M, Carmeliet P (2013) Role of PFKFB3-driven glycolysis in vessel sprouting. Cell 154:651-663

10. Yetkin-Arik B, Vogels IMC, Neyazi N, van Duinen V, Houtkooper RH, van Noorden CJF, Klaassen I, Schlingemann RO (2019) Endothelial tip cells in vitro are less glycolytic and have a more flexible response to metabolic stress than non-tip cells. Sci Rep 9:10414
11. Al Mamun A, Hayashi H, Sakima M, Sato M (2016) Adenosine triphosphate is a critical determinant for VEGFR signal during hypoxia. Am J Physiol Cell Physiol 311:C985-C995

12. Siebeneicher $H$, Cleve A, Rehwinkel H, Neuhaus R, Heisler I, Muller T, Bauser M, Buchmann B (2016) Identification and optimization of the first highly selective GLUT1 Inhibitor BAY-876. Chem Med Chem $11: 2261-2271$

13. Rufino AT, Rosa SC, Judas F, Mobasheri A, Lopes MC, Mendes AF (2013) Expression and function of K(ATP) channels in normal and osteoarthritic human chondrocytes: possible role in glucose sensing. J Cell Biochem 114:1879-1889

14. Sakima M, Hayashi H, Mamun AA, Sato M (2018) VEGFR-3 signaling is regulated by a G-protein activator, activator of G-protein signaling 8, in lymphatic endothelial cells. Cell Res, Exp. https://doi.org/10.1016/j.yexcr .2018.04.007

15. Sato M, Hiraoka M, Suzuki H, Bai Y, Kurotani R, Yokoyama U, Okumura S, Cismowski MJ, Lanier SM, Ishikawa Y (2011) Identification of transcription factor E3 (TFE3) as a receptor-independent activator of Galpha16: gene regulation by nuclear Galpha subunit and its activator. J Biol Chem 286:17766-17776

16. Hayashi H, Mamun AA, Takeyama M, Yamamura A, Zako M, Yagasaki R, Nakahara T, Kamei M, Sato M (2019) Activator of G-protein signaling 8 is involved in VEGF-induced choroidal neovascularization. Sci Rep 9:1560

17. Yamamura A, Nayeem MJ, Al Mamun A, Takahashi R, Hayashi H, Sato M (2019) Platelet-derived growth factor up-regulates Ca(2+)-sensing receptors in idiopathic pulmonary arterial hypertension. FASEB J. https:// doi.org/10.1096/fi.201802620Rfi201802620R

18. Tumova S, Kerimi A, Porter KE, Williamson G (2016) Transendothelial glucose transport is not restricted by extracellular hyperglycaemia. Vascul Pharmacol 87:219-229

19. Zhou M, Tanaka O, Sekiguchi M, He HJ, Yasuoka Y, Itoh H, Kawahara K, Abe $H$ (2005) ATP-sensitive $K+$-channel subunits on the mitochondria and endoplasmic reticulum of rat cardiomyocytes. J Histochem Cytochem 53:1491-1500

20. Marhfour I, Jonas JC, Marchandise J, Lefevre A, Rahier J, Sempoux C, Guiot Y (2010) Endoplasmic reticulum accumulation of Kir6.2 without activation of ER stress response in islet cells from adult Sur 1 knockout mice. Cell Tissue Res 340:335-346

21. Qiu L, Ding X, Zhang Z, Kang YJ (2012) Copper is required for cobaltinduced transcriptional activity of hypoxia-inducible factor-1. J Pharmacol Exp Ther 342:561-567

22. Kuang $R$, Jahangiri $A$, Mascharak S, Nguyen A, Chandra A, Flanigan PM, Yagnik G, Wagner JR, De Lay M, Carrera D, Castro BA, Hayes J, Sidorov M, Garcia JLI, Eriksson P, Ronen S, Phillips J, Molinaro A, Koliwad S, Aghi MK (2017) GLUT3 upregulation promotes metabolic reprogramming associated with antiangiogenic therapy resistance. JCI Insight 2:e88815

23. Baumann MU, Zamudio S, Illsley NP (2007) Hypoxic upregulation of glucose transporters in BeWo choriocarcinoma cells is mediated by hypoxia-inducible factor-1. Am J Physiol Cell Physiol 293:C477-C485

24. Wieman HL, Wofford JA, Rathmell JC (2007) Cytokine stimulation promotes glucose uptake via phosphatidylinositol-3 kinase/Akt regulation of Glut1 activity and trafficking. Mol Biol Cell 18:1437-1446

25. Paik JY, Lee KH, Ko BH, Choe YS, Choi Y, Kim BT (2005) Nitric oxide stimulates 18F-FDG uptake in human endothelial cells through increased hexokinase activity and GLUT1 expression. J Nucl Med 46:365-370

26. Zhu L, Wu G, Yang X, Jia X, Li J, Bai X, Li W, Zhao Y, Li Y, Cheng W, Liu S, Jin $S$ (2019) Low density lipoprotein mimics insulin action on autophagy and glucose uptake in endothelial cells. Sci Rep 9:3020

27. Egert S, Nguyen N, Schwaiger M (1999) Myocardial glucose transporter GLUT1: translocation induced by insulin and ischemia. J Mol Cell Cardiol 31:1337-1344

28. Perrini S, Natalicchio A, Laviola L, Belsanti G, Montrone C, Cignarelli A, Minielli V, Grano M, De Pergola G, Giorgino R, Giorgino F (2004) Dehydroepiandrosterone stimulates glucose uptake in human and murine adipocytes by inducing GLUT1 and GLUT4 translocation to the plasma membrane. Diabetes 53:41-52

29. Calado SM, Alves LS, Simao S, Silva GA (2016) GLUT1 activity contributes to the impairment of PEDF secretion by the RPE. Mol Vis 22:761-770

30. Bae SS, Cho H, Mu J, Birnbaum MJ (2003) Isoform-specific regulation of insulin-dependent glucose uptake by Akt/protein kinase B. J Biol Chem 278:49530-49536 
31. Meireles P, Sales-Dias J, Andrade CM, Mello-Vieira J, Mancio-Silva L, Simas JP, Staines HM, Prudencio M (2017) GLUT1-mediated glucose uptake plays a crucial role during Plasmodium hepatic infection. Cell, Microbiol, p 19

32. Foster MN, Coetzee WA (2016) KATP channels in the cardiovascular system. Physiol Rev 96:177-252

33. Clement JP, Kunjilwar K, Gonzalez G, Schwanstecher M, Panten U, AguilarBryan L, Bryan J (1997) Association and stoichiometry of KATP channel subunits. Neuron 18(5):827-838

34. Mikhailov MV, Campbell JD, de Wet H, Shimomura K, Zadek B, Collins RF, Sansom MS, Ford RC, Ashcroft FM (2005) 3-D structural and functional characterization of the purified KATP channel complex Kir6.2-SUR1. EMBO J 24:4166-4175
35. McTaggart JS, Clark RH, Ashcroft FM (2010) The role of the KATP channel in glucose homeostasis in health and disease: more than meets the islet. J Physiol 588:3201-3209

36. Quesada I, Rovira JM, Martin F, Roche E, Nadal A, Soria B (2002) Nuclear KATP channels trigger nuclear $\mathrm{Ca}(2+)$ transients that modulate nuclear function. Proc Natl Acad Sci USA 99:9544-9549

\section{Publisher's Note}

Springer Nature remains neutral with regard to jurisdictional claims in published maps and institutional affiliations.
Ready to submit your research? Choose BMC and benefit from:

- fast, convenient online submission

- thorough peer review by experienced researchers in your field

- rapid publication on acceptance

- support for research data, including large and complex data types

- gold Open Access which fosters wider collaboration and increased citations

- maximum visibility for your research: over 100M website views per year

At BMC, research is always in progress.

Learn more biomedcentral.com/submissions 